\title{
ESTRUTURA INTELECTUAL DA PESQUISA EM EAD NO CENÁRIO MUNDIAL
}

\author{
RIBEIRÃO PRETO/SP JUNHO/2018 \\ José Dutra de Oliveira Neto - USP - dutra@usp.br \\ André Marino de Paiva - USP - dutra@usp.br \\ Tipo: Investigação Científica (IC) \\ Natureza: Descrição de Projeto em Andamento \\ Categoria: Pesquisa e Avaliação \\ Setor Educacional: EDUCAÇÃO SUPERIOR
}

\begin{abstract}
RESUMO
A incorporação de novos indicadores da pesquisa em Educação à Distância (EAD) pode resultar em informações mais precisas para avaliar a evolução da área. Diversas pesquisas têm sido publicadas sem, no entanto, mostrar um modelo validado para a pesquisa científica em EaD. Este estudo avalia um modelo existente utilizado para avaliar a estrutura intelectual da pesquisa em Educação a Distância. Uma nova amostra de 1868 artigos, agora incluindo cinco revistas internacionais de Educação a Distância, no período de 2000 até 2017, foram coletados e analisados sob a perspectiva da ciência normal proposta por Khun. Este modelo poderá auxiliar as futuras estratégias de pesquisa para as universidades.
\end{abstract}

Palavras-chave: Pesquisa em EAD

\section{AGRADECIMENTOS}

A FAPESP 


\section{?1.Introdução}

Uma das formas de avaliação da evolução de uma área de conhecimento é por meio da revisão da literatura. Seus resultados podem impactar no processo de ensino e aprendizagem e colaborar com novas estratégias educacionais (Gall, Gall, \& Borg, 2007). Estas pesquisas de revisão são presentes na literatura e algumas geram modelos que permitem prever o comportamento da pesquisa em uma área de conhecimento, mas sem no entanto provocar reações de desconfiança da comunidade acadêmica (Vinkler, 2010).Muitos trabalhos já foram publicados sobre o tema (Holmberg, 1985), (Scriven, 1991), (Panda, 1992), (Suen \& Stevens, 1993). ), (Jegede, 1994), (Koble \& Bunker, 1997), (Mishra, 1997), (Berge \& Mrozowski, 2001), (Rourke \& Szabo, 2002), (Lee, Driscol, \& Nelson, 2004), ( Tallent-Runnels, Thomas, Lan e Cooper, 2006), (Lee, Driscol, \& Nelson, 2007), (Zawacki-Richter, 2009), (Zawacki-Richter, Backer, \& Vogt, 2009) (Oliveira Neto e Santos , 2010). Todas estas pesquisas propõe uma nova avaliação segundo novas variáveis, sem no entanto, propor um modelo validado para a interpretação da produção científica em EaD. Apenas o modelo proposto por Oliveira Neto (2012) tem esta pretensão de desenvolver um novo modelo, porém ainda sem a validação final.

O foco deste estudo deriva deste novo modelo considerando um novo ciclo de 16 anos, além da inclusão de uma nova revista acadêmica. Ao analisar um novo ciclo, acreditamos em poder colaborar para a validação do referido modelo.

O objetivo deste estudo é validar, por meio de uma nova amostra, contribuir para a validação do modelo para analisar a produção científica no campo da Educação a distância.

\section{Referencial Teórico}

Analisaremos a produção científica da Educação a Distância sob as seguintes dimensões.

\section{Dimensão 1: Padrão Geral de Produção Científica}

A primeira dimensão se refere as características dos artigos produzidos em um determinado período e representa um conjunto de macro-indicadores sobre uma área de conhcimento. Como resultado desta dimensão, temos indicadores gerais da produção científica no campo da Educação a Distância. Com base nesta discussão propõe-se a primeira hipótese $(\mathrm{H} 1)$ : Qual é o padrão geral de produção científica no campo de 
Educação a Distância na amostra estudada?

\section{Dimensão 2: Acumulação do Conhecimento}

Duas dimensões serão computadas para medir o nível de codificação do conhecimento no campo de DE e determinar se qualquer dado estudo sobre Educação a Distância está alinhado com o que Kuhn atribui a uma ciência normal: acumulação de conhecimento e identificação e análise de literatura básica no campo (como citado em Just, Perrey, \& Meyer, 2010). Estes indicadores permitem uma análise que evidenciem a transferência de conhecimento de uma área. A segunda dimensão se refere ao que Merton definiu como "obliteração por incorporação (apud Just et al., 2010). Indica a substituição de referências mais antigas por novas referências mais recentes sobre o tema (Garfield, 1975). Quanto mais codificado o campo do conhecimento, maior é a sua taxa de obliteração (Cole, 1983). Uma ciência considerada normal deve ter uma idade média baixa de citações e evidenciar o uso de referências mais recentes. Com base nesta discussão, a segunda hipótese $(\mathrm{H} 2)$ é proposta: A pesquisa de Educação a Distância mostra evidências do fenômeno de "obliteração por incorporação"?

\section{Dimensão 3: Literatura Principal (Core)}

A partir da definição do conhecimento básico e conhecimento de fronteira proposto por Cole(1983) vamos identificar os principais artigos citados que correspondem a um conjunto estável de teorias consolidadas. Isto contrapõe com a literatura de fronteira que corresponde a novas propostas em que apenas parte se torna a literatura principal (core).

Com base nesta discussão, propõe-se a terceira hipótese (H3): Em que nível é encontrada uma forte e persistente concentração de citações para várias referências referentes à literatura core sobre pesquisa na Educação a Distância?

\section{Dimensão 4: Estrutura Intelectual}

O modelo proposto por Kuhn foi considerado para avaliar a estrutura intelectual do conhecimento. Ele propôs a substituição do conceito de ciência como uma atividade exclusivamente racional, categorizando a ciência em duas fases: ciência normal e ciência revolucionária.

Com base na discussão acima, propõe-se a quarta hipótese $(\mathrm{H} 4)$ : A estrutura intelectual é representada por unidades interdependentes de análise com um conjunto de 
referências centrais estáveis complementadas por um fluxo contínuo de novos estudos para o mesmo paradigma?

\section{Procedimentos metodológicos}

\section{População, Amostra, Período e Categorização}

Os seguintes critérios foram empregados para selecionar periódicos de EAD para este estudo: (1) mínimo de duas edições por ano; (2) existência mínima de 15 anos; (3) tendo sido publicado de 2000 a 2017; (4) acesso total aos artigos quando a categorização não era viável apenas do resumo, e (5) artigos disponíveis em inglês. Isto resultou em 5 periódicos em que conseguimos acesso por meio da instituição de pesquisa a qual o pesquisador pertence. Isto resultou em 1868 artigos (Quadro 1).

As citações foram extraídas do Google Scholar® (versão em inglês) a partir de cada um dos 1868 artigos coletados.

\begin{tabular}{|l|l|l|l|}
\hline Periódico & Vol 1 & $\begin{array}{l}\text { Ultimo } \\
\text { Vol }\end{array}$ & \# Artigos \\
\hline $\begin{array}{l}\text { A.JDE } \\
\text { (USA, desde 1987) }\end{array}$ & $14-1$ & $31-2$ & 259 \\
\hline $\begin{array}{l}\text { International Journal of E-Learning \& Distance Education } \\
\text { (antes de 29-1(2014) JDE) } \\
\text { (Canada, desde 1986) }\end{array}$ & $15-1$ & $32-2$ & 210 \\
\hline $\begin{array}{l}\text { DE } \\
\text { (Australia, desde 1980) }\end{array}$ & $21-1$ & $38-3$ & 343 \\
\hline $\begin{array}{l}\text { OL } \\
\text { (England, desde 1986) }\end{array}$ & $15-1$ & $32-3$ & 291 \\
\hline $\begin{array}{l}\text { International Review of Research in Open and Distance } \\
\text { Learning } \\
\text { (desde 2000) }\end{array}$ & $1-1$ & $18-7$ & 765 \\
\hline Total & & & 1868 \\
\hline
\end{tabular}

\section{Categorização}

A tipologia empregada para categorizar os 1868 artigos em unidades de análise é composta por: Projeto, Desenvolvimento, Gestão, Avaliação, Operações e Teoria e Pesquisa (Lee et al., 2007). Isto permitirá a comparação dos resultados com pesquisas anteriores cujo período antecede esta pesquisa.

Em seguida, os perfis dos pesquisadores foram analisados à luz da categoria 
Produtividade, que classifica os autores em continuantes, recém-chegados, transitórios, terminais e One-timers (Braun, Glanzel \& Schubert, 2001; Guarido Filho, 2008).

\section{Procedimentos de Coleta de Dados}

Após o acesso aos artigos originais, foi elaborada uma planilha Excel® contendo informações sobre as seguintes características dos artigos: número do artigo original, nome do periódico, ano de publicação, título, resumo, citações, autores e período (P1 . P2, P3 e P4), onde cada período compreende 4 anos.

\section{Dimensão 1 - Produção Científica Geral}

Primeiramente, foram coletadas as informações descritivas sobre o número de referências, número de autores e citações para cada ciclo de 4 anos. Uma análise global e longitudinal foi realizada para investigar possíveis mudanças nos diversos ciclos. Posteriormente identificamos líderes, indicadores de produtividade e continuidade dos autores.

\section{Dimensão 2 - Acumulação de Conhecimento}

Essa dimensão foi calculada a partir da idade das referências encontradas nos artigos da amostra durante o período de 16 anos sob investigação. O ano de publicação do periódico e o ano de publicação das referências, foram utilizados para este cálculo.

\section{Dimensão 3 - Literatura Básica (core)}

Os trinta artigos mais citados nas referências constituíram a literatura core sobre Educação a Distância.

\section{Dimensão 4 - Estrutura Intelectual}

A análise das dimensões 3 e 4 mostram informações baseadas em tempo sobre a eficiência da transferência de conhecimento. A estrutura intelectual é representada por unidades de análise interdependentes, com um conjunto de referências centrais estáveis complementadas por um influxo de novas pesquisas sob o mesmo paradigma. A unidade de análise utilizada neste estudo foi a categorização dos tópicos de pesquisa propostos por Lee et al. (2007).

\section{Referências}


Berge, Z. L., \& Mrozowski, S. (2001). Review of research in distance education, 1990 to 1999. American Journal of Distance Education, 15(3), 5-19.

Braun, T., Glanzel, W., \& Schubert, A. (2001). Publication and cooperation patterns of the authors of neuroscience journals. Scientometrics, 51(3), 499-510.

Cole, S. (1983). The Hieraqchy of the Sciences? The American Journal of Sociology,(1), 111-139.

Cole, S. (1992). Making Science Between Nature and Society. Cambridge: Harvard University Press.

Ding, W. W., Levin, S. S., Stephan, P. E., \& Winkler, A. E. (2010). The impact of Information technology on academic scientists' productivity and collaborations patterns. Management Science, 56(9), 1439-1461.

Gall, J. P., Gall, J. P., \& Borg, W. R. (2007). Educational research: An introduction Pearson.

Garfield, E. (1975). The "obliteration phenomenon" in science - and the advantage of being obliterated. Current Contents, 22, -5 .

Guarido Filho, E. R. (2008). A construção da teoria institucional nos estudos organizacionais no Brasil: O período de 1993-2007 (, Universidade Federal do Paraná, Curitiba).

Holmberg, B. (1985). Status and trends of distance education. Lund: Lector Publishing.

Jegede, O. J. (1994). Distance education research priorities for Australia: A study of the opinions of distance educators and practitioners. Distance Education, 15(2), 234-253.

Just, A., Perrey, E., \& Meyer, M. (2010). Accounting as a Normal Science? - An Empirical Investigation of the Intellectual Structure of Accounting Research. American Accounting Association.

Koble, M. A., \& Bunker, E. L. (1997). Trends in research and practice: An examination of The American Journal of Distance Education 1987 to 1995. American Journal of Distance Education, 11(2), 19-38. 
Kuhl, T. S. (1996). The Structure of Scientific Revolution (3 ed.). Chicago: The University of Chicago Press.

Lee, Y., Driscol, M. P., \& Nelson, W. (2004). The past, present, and future of research in distance education: Results of a content analysis. American Journal of Distance Education, 18(4), 225-241.

Lee, Y., Driscol, M. P., \& Nelson, W. (2007). Trends in research: A content analysis of major journals. In M.G. Moore (Ed.), Handbook of distance education (pp. 31-41). Mahwah: Lawrence Erlbaum Associates.

Mishra, S. (1997). A critical analysis of periodical literature in distance education. Indian Journal of Open Learning, 6(1), 39-54.

Oliveira Neto, J.D. Avaliação da pesquisa em EAD baseada no modelo da ciência normal, In: 18 Congresso Internacional de Educação a Distância,São Luis, 2012

Panda, S. (1992). Distance Educational reserach in India: Stock-taking, concerns and prospects. Distance Education, 13(2), 309-326. doi:10.1080/0158791920130211

Rourke, L., \& Szabo, M. A. (2002). A content analysis of the Journal of Distance Education 1986-2001. Available from Journal of Distance Education. (17).

Scriven, B. (1991). Ten years of 'Distance Education'. Distance Education, 12(1), 137-153.

Suen, H. K., \& Stevens, R. J. (1993). Analytic consideration in distance education research. American Journal of Distance Education, 7(3), 61-69. doi:10.1080/08923649309526834

Tallent-Runnels, M. K., Thomas, J. A., Lan, W. Y., \& Cooper, S. (2006). Teaching courses online: A review of the research. Available from Review of Educational Research. (76).

Vinkler, P. (2010). The evaluation of Reseach by Scientometrics Indicators (1 ed.) Chandos Publishing.

Ward, A. W., Hall, B. W., \& Schram, C. F. (1975). Evaluation of published educational research: A national survey. American Educational Research Journal, 12, 109-128. 
Zawacki-Richter, O. (2009). Research areas in distance education: A Delphi study. International Review of Research in Open and Distance Learning, 10(3).

Zawacki-Richter, O., Backer, E. M., \& Vogt, S. (2009). Review of Distance Education Research (2000 to 2008): Analysis of Reseacrh Areas, Methods, and Autorship Patterns. International Review of Research in Open and Distance Learning, 10(6). 